\title{
Neural mechanisms of individual differences in temporal discounting of monetary and primary rewards in adolescents
}

\author{
Erik de Water ${ }^{\mathrm{a}, *}$, Gabry W. Mies ${ }^{\mathrm{a}}$, Bernd Figner ${ }^{\mathrm{a}}$, Yuliya Yoncheva ${ }^{\mathrm{b}}$, Wouter van den Bos ${ }^{\mathrm{c}}, \mathrm{F}$. \\ Xavier Castellanos ${ }^{\mathrm{b}, \mathrm{d}}$, Antonius H.N. Cillessen ${ }^{\mathrm{a}}$, Anouk Scheres ${ }^{\mathrm{a}}$ \\ a Behavioural Science Institute, Radboud University, Nijmegen, The Netherlands \\ b Department of Child and Adolescent Psychiatry, Center for Neurodevelopmental Disorders, NYU Langone Medical Center, New York, NY, USA \\ c Center for Adaptive Rationality, Max Planck Institute for Human Development, Berlin, Germany \\ d Nathan Kline Institute for Psychiatric Research, Orangeburg, NY, USA
}

\section{A R T I C L E I N F O}

\section{Keywords:}

Adolescents

Temporal discounting

fMRI

Individual differences

\begin{abstract}
A B S T R A C T
Adolescents are generally characterized as impulsive. However, impulsivity is a multi-dimensional construct that involves multiple component processes. Which of these components contribute to adolescent impulsivity is currently unclear. This study focused on the neural mechanisms underlying individual differences in distinct components of temporal discounting (TD), i.e., the preference for smaller immediate rewards over larger delayed rewards. Participants were 58 adolescents (12-16 years-old) who performed an fMRI TD task with both monetary and snack rewards. Using mixed-effects modeling, we determined participants' average impatience, and further decomposed TD choices into: 1) amount sensitivity (unique contribution of the magnitude of the immediate reward); and 2) delay sensitivity (unique contribution of delay duration). Adolescents' average impatience was positively correlated with frontoparietal and ventral striatal activity during delayed reward choices, and with ventromedial prefrontal cortex activity during immediate reward choices. Adolescents' amount sensitivity was positively associated with ventral striatal and dorsal anterior cingulate cortex activity during immediate reward choices. Delay sensitivity was positively correlated with inferior parietal cortex activity during delayed reward choices. As expected, snacks were discounted more steeply than money, and TD of both reward types was associated with overlapping activation in the inferior parietal cortex. Exploring whether testosterone or estradiol were associated with TD and its neural correlates revealed no significant associations. These findings indicate that distinct components contribute uniquely to TD choice and that individual differences in amount sensitivity are uniquely associated with activation of reward valuation areas, while individual differences in delay sensitivity are uniquely associated with activation of cognitive control areas.
\end{abstract}

\section{Introduction}

Adolescents are typically characterized as impulsive. For instance, compared to children and adults, they show increased levels of substance use and other reckless behaviors (Steinberg, 2008). However, not all adolescents are equally impulsive. Importantly, adolescents who are highly impulsive are at a heightened risk to develop behavioral problems, such as substance abuse (Audrain-McGovern et al., 2009), with substantial costs across all domains of life (i.e., social, financial, health) (Mertens et al., 2003). Therefore, it is important to understand the mechanisms underlying individual differences in adolescent impulsivity, as this could inform prevention and intervention programs.

A key component of impulsivity, namely the preference for smaller, more immediate rewards over larger delayed ones has been widely studied with temporal discounting (TD) tasks (see Scheres et al. (2013), for a review). TD refers to the decrease in subjective value of a reward as the delay preceding its delivery is increased. TD tasks involve choices between smaller, more immediate rewards (e.g., $\$ 2$ today) and larger, delayed rewards (e.g., \$10 in 90 days). Adolescents with psychiatric disorders whose core symptom includes impulsivity (e.g., AttentionDeficit/Hyperactivity Disorder (ADHD), substance abuse, gambling, and conduct disorders) have been found to show steeper TD than typically developing adolescents (Demurie et al., 2012; Jackson and MacKillop, 2016; MacKillop et al., 2011; Patros et al., 2016; Reynolds, 2006; Scheres et al., 2010; White et al., 2014). Typically developing adolescents also show steeper TD than adults (de Water et al., 2014; Olson et al., 2007; Scheres et al., 2006; Steinberg et al., 2009; van den Bos et al., 2015; but see Scheres et al. (2014)).

\footnotetext{
* Correspondence to: Behavioural Science Institute, Radboud University, Montessorilaan 3, 6500 HE Nijmegen, The Netherlands.

E-mail address: erikdewater@gmail.com (E. de Water).
} 
Studies investigating the neural correlates of TD have implicated both frontoparietal and limbic brain areas in TD, including the lateral prefrontal cortex (PFC), parietal cortex, ventral striatum (VS), medial PFC (mPFC) and posterior cingulate cortex (PCC) (Scheres et al., 2013). The few studies that have explored the neural mechanisms of individual differences in TD in adolescents (Benningfield et al., 2014; Ripke et al., 2012; Stanger et al., 2013) have found that differential functioning of both frontoparietal and limbic areas contributes to these individual differences. One study reported that adolescents who exhibit relatively steep TD show increased activation of lateral prefrontal and parietal brain areas during delayed reward choices, and increased activation of the VS and ventromedial PFC (vmPFC) during immediate reward choices (Stanger et al., 2013). However, other studies reported decreased VS activation during decision-making (Ripke et al., 2012) or reward processing (Benningfield et al., 2014) in adolescents who show relatively steep TD.

In the present study, we used mixed-effects modeling (Baayen et al., 2008) to determine individual differences in adolescents' average preference for immediate rewards (i.e., average impatience) and two distinct components of TD choice: 1) amount sensitivity, or the unique effect of the amount of the immediate reward; and 2) delay sensitivity, or the unique effect of the delay duration. The first goal of this study was to investigate the neural mechanisms underlying individual differences in adolescents' average impatience, and amount and delay sensitivity. We hypothesized that average impatience would be positively associated with superior parietal cortex activity (Stanger et al., 2013), and either positively (Stanger et al., 2013) or negatively (Figner et al., 2010; Gianotti et al., 2012) associated with dorsolateral PFC (DLPFC) activity during delayed reward choices. Further, we predicted that average impatience would be positively associated with activation of the vmPFC (McClure et al., 2004; Stanger et al., 2013) and with differential recruitment of the VS (Benningfield et al., 2014; Ripke et al., 2012) during immediate reward choices. We expected that amount sensitivity would be positively associated with VS and vmPFC activity during immediate reward choices (Kable and Glimcher, 2007; van Duijvenvoorde et al., 2015). We anticipated that delay sensitivity would be positively associated with amygdala and insula activation during delayed reward choices (Lemiere et al., 2012; Plichta et al., 2009; Wilbertz et al., 2013).

The second goal of this study was to directly compare the neural correlates of TD of money and snacks in adolescents, and to explore whether amount and delay sensitivity contributed differently to TD of both reward types. We expected that adolescents would discount delayed snack rewards more steeply than monetary rewards
(Demurie et al., submitted for publication; Estle et al., 2007; Jimura et al., 2011). We also expected that TD choices for money and snacks would both activate frontoparietal regions (McClure et al., 2004, 2007), and that monetary choices would activate the medial orbitofrontal cortex (mOFC) more than snack choices, whereas snack choices would activate the anterior insula more than money choices (Sescousse et al., 2013).

The third goal of this study was to explore whether testosterone and estradiol levels might be associated with TD components and their neural correlates. When investigating the neural mechanisms underlying individual differences in TD in adolescence, it is important to account for pubertal hormone levels (e.g., testosterone and estradiol), as they increase rapidly in adolescence (Peper and Dahl, 2013), and have been reported to stimulate impulsive behaviors (de Water et al., 2013) by influencing brain activity in reward-related brain areas, such as the VS (Braams et al., 2015; Op de Macks et al., 2011). We expected that higher testosterone and estradiol levels would be associated with increased discounting of delayed rewards (Bromberg et al., 2015), and increased VS activation during decision-making (Braams et al., 2015; Op de Macks et al., 2011).

\section{Methods}

\section{Participants}

Participants were 61 adolescents (all but one right-handed) who completed a functional magnetic resonance imaging (fMRI) session. Three participants were excluded from subsequent analyses, due to head motion $>3 \mathrm{~mm}$, a brain anomaly, or limited field-of-view coverage. Thus, data from 58 adolescents (31 girls; $M$ age $=14.5$ years, $S D=1.2$, range $=12-16$ years $)$ were reported. IQ $(M=109, S D=13$, range 80-135) was estimated from the Vocabulary and Block Design subtests of the WISC-III (Wechsler, 1991). The Child Behavior Checklist (CBCL; Achenbach and Rescorla, 2001) was filled out by a parent. No participant scored in the clinical range ( $\mathrm{T}$-score $\geq 70$ ) for internalizing or externalizing problems.

\section{Temporal discounting task}

Each participant performed a TD task in the MRI scanner (see Fig. 1). The TD task included two blocks with monetary rewards, and two blocks with snack rewards. The task was potentially real, in that one choice was randomly selected for each reward type and given to participants. If the participant chose an immediate reward, they received the respective outcome (money and snack) immediately. If

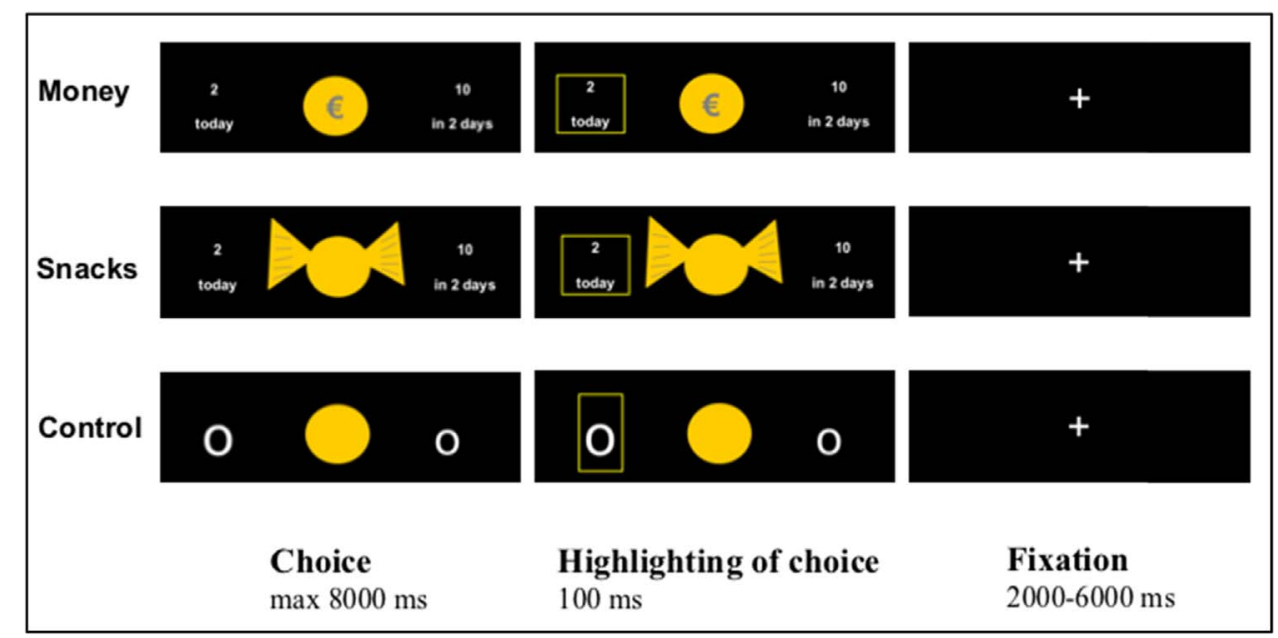

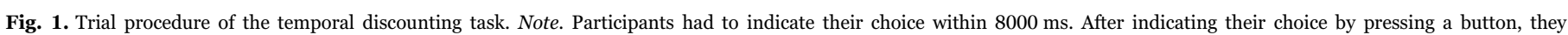
immediately continued to the variable fixation period before the next trial. 
the participant chose a delayed reward, the money was transferred to their bank account, and the snack was mailed or delivered to their home at the corresponding delay. A potentially real task relies on the assumption that each choice will be made as if it has real consequences, since participants do not know which choice will be selected and paid (Scheres et al., 2013).

A total of 80 choices were administered for each reward type between a small reward available on the day of testing ("today"), and a larger reward available after a delay. In snack trials, participants chose between different amounts of their favorite snack, selected from a list of 14 types (see Supplementary Table 1). The delayed reward was always $€ 10$ or 10 snacks for the monetary or snack task, respectively. The immediate reward was $\square 2, \square 4, \square 6$, or $\square 8$ or $2,4,6$ or 8 snacks. There were five delays: $2,14,30,90$, and 180 days. For each trial type, each immediate reward-delay combination was presented four times. In addition to money and snack choices, there were 20 control trials in which participants chose the larger of two circles. These control trials allowed us to compare brain activity between TD choices and choices not requiring consideration of reward amounts or delays but only a perceptual decision and motor response.

Participants indicated their preference by pressing a button on a button box with their right index finger (for the option presented on the left) or their right middle finger (for the option on the right). Participants were given $8000 \mathrm{~ms}$ to indicate their preference, and their choice was highlighted for $100 \mathrm{~ms}$, followed by the presentation of a fixation cross during the intertrial interval with a variable duration (2000-6000 ms; $M=3656$ ms) (cf. Ballard and Knutson, 2009; Bickel et al., 2009; Peters and Buechel, 2009, 2010; Weber and Huettel, 2008) to jitter the trial onsets. The position of the options on the screen was counterbalanced across trials.

Each participant completed 22 practice trials (10 money choices, 10 snack choices, and 2 control choices) before entering the scanner. In the scanner, the TD task was administered in 4 blocks of 45 choices ( 40 TD choices and 5 control choices). Money and snack choices were administered in four separate, alternating blocks (counterbalanced across participants). The TD task lasted approximately $20 \mathrm{~min}$.

Participants were asked to refrain from eating snacks $24 \mathrm{~h}$ before the experiment. The experimenter weighed and measured each participant in order to compute their Body Mass Index (BMI; weight in $\mathrm{kg}$ / height in $\mathrm{cm}^{2}$ ). Participants also reported the time since their last meal (in hours and minutes), and how hungry they felt on a 10-point scale, immediately before the TD task. None of these variables correlated significantly (all $p$ 's > .12) with TD choices (neither money nor snack choices).

\section{Reward valuation}

Participants rated on a 10-point scale how much they would enjoy receiving each reward that was used in the task (see Fig. S1). Participants' average rating across the rewards of each type (money, snacks) was taken as an index of subjective reward valuation of that reward type. Participants also indicated how much money they were willing to pay for each snack amount (see Fig. S2). These reward valuation questions were administered before the task for half the participants, and after the task for the other half.

\section{Subjective delay perception}

We assessed participants' subjective delay perception by presenting them with a $180 \mathrm{~mm}$ visual analogue scale, with the anchors "very short" and "very long" below the left and right ends of the scale, respectively (Zauberman et al., 2008). For each delay used in the task, participants indicated how long they considered it by placing a pencil mark on the line. The length (in $\mathrm{mm}$ ) from the left end of the line to the mark was taken as an index of subjective delay perception (see Fig. S3).

\section{Quick delay questionnaire}

To assess participants' delay aversion and delay discounting in daily life, they filled out the Quick Delay Questionnaire (QDQ; Clare et al., 2010; Hsu et al., 2015), which has high test-retest reliability $(r=.81)$. QDQ delay aversion and delay discounting scores positively correlate with ADHD symptoms (Clare et al., 2010). The QDQ has 5 items for delay aversion (e.g., "Having to wait for things makes me feel stressed and tense "), and 5 items for delay discounting (e.g., "The future is not important to me, I only consider the immediate consequences of my actions"); participants rated how well each statement described their behavior in the past 6 months on a 5 -point scale $(1=$ not like me at all, $5=$ very like me). The five ratings for each scale were averaged to one for delay aversion (Cronbach's $\alpha=.78$ ) and delay discounting ( $\alpha=.61$ ). Scores for positively phrased questions were reversed, such that higher scores indicated more delay aversion or discounting.

\section{Pubertal hormones}

Participants collected saliva samples by passive drool at home on two consecutive days. Girls who had reached menarche collected the samples on days 6 and 7 after the start of their period, or during their stop week if they used oral contraceptives $(n=1)$ (see de Water et al. (2013), Peper et al. (2013)). On each day, participants collected two saliva samples: directly after waking up, and 15 min later. The two daily samples were first combined (Wood, 2009), and testosterone and estradiol levels were subsequently determined using an enzyme-linked immunosorbent assay (ELISA; IBL, Hamburg, Germany) at the Clinical Chemistry Lab of Medical Centre Alkmaar, the Netherlands. The lowest detectable levels were $0.4 \mathrm{pg} / \mathrm{mL}$ for estradiol, and $4.7 \mathrm{pg} / \mathrm{mL}$ for testosterone. The reliability coefficients of the hormone assays are reported in Supplementary materials.

Given that hormone levels for the two days were highly correlated (testosterone: $r h o=.77, p<.001$; estradiol: $r h o=.55, p<.001$ ), we used average testosterone and estradiol levels for the two days in analyses. Testosterone level was log-transformed to reduce positive skew. One outlier ( $>3 \mathrm{SD}$ of the mean within each gender) was excluded from analyses (1 boy for estradiol).

Participants completed the Pubertal Development Scale (PDS; Petersen et al., 1988), to report Tanner stages (Carskadon and Acebo, 1993). Consistent with prior studies with participants of the same age (Crockett and Petersen, 1987), all Tanner stages were represented in our sample: prepubertal (6.7\%), early pubertal (10.0\%), midpubertal (31.7\%), late pubertal (48.3\%), and postpubertal (3.3\%). As expected, girls reported more advanced Tanner stages than boys $(t=2.95, p=.005)$, even though they did not differ significantly in age $(t=0.63, p=.53)$.

\section{fMRI data collection}

Neuroimaging data were collected using a 1.5T Siemens Avanto scanner with a 32-channel head coil. To minimize head movement, we placed foam inserts around each participants' head and paper tape across their forehead and the head coil. We collected: (1) a T1-weighted anatomical scan (repetition time $=2250 \mathrm{~ms}$, echo time $=2.95 \mathrm{~ms}$, field of view $=256 \mathrm{~mm}, 176$ slices, slice thickness $=1 \mathrm{~mm}$, slice gap $=0.5 \mathrm{~mm}$, flip angle $=15^{\circ}$, duration $=5 \min 14 \mathrm{~s}$ ), and (2) functional images using a multi-echo GRAPPA sequence during four runs of approximately 5 min each (repetition time $=2010 \mathrm{~ms}$, echo times $=9.4,20.9,33,44$, and $56 \mathrm{~ms}$, field of view $=224 \mathrm{~mm}, 32$ sequential slices collected in ascending order, slice thickness $=3 \mathrm{~mm}$, slice gap $=0.51 \mathrm{~mm}$, flip angle $=90^{\circ}$ ). Before the first run, we collected 30 volumes (prescans). The first two volumes of runs 2-4 were discarded to allow for steady state magnetization. 


\section{Procedure}

Participants were first familiarized with the scanner environment by performing practice trials of the TD task in a mock scanner. In the MRI scanner, they first watched a movie while the T1-weighted anatomical scan was collected, followed by the TD task. A second task (social exclusion paradigm) and a DTI scan were collected after the TD task, which are not reported in this manuscript. After the MRI session, participants completed the QDQ, PDS and WISC-III subtests. Participants were paid $€ 20$ for their participation, and could earn an additional $€ 2-€ 10$ and $2-10$ snacks depending on the randomly selected choices in the TD task. Parents gave informed consent, participants gave informed assent. All procedures were approved by the Medical Ethical Committee of the first author's institute.

\section{Mixed-effects model analyses}

TD task choices were analyzed with a generalized linear mixedeffects model using the lme4 package (Bates et al., 2015) in R (version 3.2.0; $\mathrm{R}$ Core Team, 2013). The dependent variable was TD choice ( $1=$ immediate reward choice, $0=$ delayed reward choice) (see Fig. 2 for individual differences in the \% of delayed reward choices). The model included a fixed intercept and the following fixed effects: amount of the immediate reward, length of the delay, reward type (snacks, money), the interaction between amount and reward type, and the interaction between delay and reward type. In addition, for each participant, random adjustments to the fixed intercept and to the slopes of all fixed effects (main effects and interactions) were included. Furthermore, all correlation terms among the random effects were estimated, resulting in a model "maximal with respect to the random effects" as suggested by Barr et al. (2013), to avoid inflated Type 1 errors. Amount and delay were standardized (centered and scaled), and reward type was specified by a sum-to-zero contrast (1=snacks, $-1=$ money). The model was estimated using the objective amounts and delays, as this model fit better than the model with subjective amounts and delays based on participants' reward valuation and subjective delay perception ratings (see Supplementary materials).

The fitted mixed-effects model reads in formal notation:

First level: logit $\left(y_{i t}\right)=\pi_{O i}+\pi_{1 i} *$ Amount $_{\mathrm{t}}+\pi_{2 i} *$ Delay $_{\mathrm{t}}+\pi_{3 i} *$ Reward Type $_{\mathrm{t}}+\pi_{4 i} *\left(\right.$ Reward Type $_{\mathrm{t}} *$ Amount $\left._{\mathrm{t}}\right)+\pi_{5 i} *$ (Reward Type $_{t} *$ Delay $\left._{\mathrm{t}}\right)+\varepsilon_{i t}$

Second level:

$\pi_{0 \mathrm{i}}=\gamma_{00}+\xi_{0 \mathrm{i}}$

$\pi_{1 \mathrm{i}}=\gamma_{10}+\xi_{1 \mathrm{i}}$

$\pi_{2 \mathrm{i}}=\gamma_{20}+\xi_{2 \mathrm{i}}$

$\pi_{3 \mathrm{i}}=\gamma_{30}+\xi_{3 \mathrm{i}}$

$$
\pi_{4 \mathrm{i}}=\gamma_{40}+\xi_{4 \mathrm{i}}
$$$$
\pi_{5 \mathrm{i}}=\gamma_{50}+\xi_{5 \mathrm{i}}
$$

In which $y_{i t}$ indicates the response of the $i$ th individual at the $t$ th trial, with $y_{i t}=0$ denoting a delayed reward choice and $y_{i t}=1$ denoting an immediate reward choice. Substitution of the second level model into the first level model gives the integrated model that was fitted to the data. Parameters in this model are the fixed effects $(\gamma)$ and the random effects (the variance of $\varepsilon$ term and $\xi$ terms). The variance $\xi_{O i}$ denotes between-participant variance in the tendency to select the immediate reward (average impatience), and the variances $\xi_{1 i}$ and $\xi_{2 i}$ denote between-participant variance in the effect of amount (amount sensitivity) and delay (delay sensitivity) on immediate reward choice, respectively. The variance $\xi_{3 i}$ denotes between-participant variance in the effect of reward type on immediate reward choice, and the variances $\xi_{4 i}$ and $\xi_{5 i}$ denote between-participant variance in the reward type * amount interaction and the reward type * delay interaction, respectively. Finally, the variance $\varepsilon_{i t}$ denotes within-participant variance. As delay and amount were standardized, the fixed intercept, $\gamma_{00}$, denotes the tendency to select the immediate reward for average amount and delay. The model also includes main effects of amount and delay $\left(\gamma_{10}\right.$ and $\gamma_{20}$, respectively), a main effect of reward type $\left(\gamma_{30}\right)$, as well as interactions of reward type with amount and delay $\left(\gamma_{40}\right.$ and $\gamma$ ${ }_{50}$, respectively). All fixed and random effects estimate how a change in one of the predictors (e.g., the effect of amount on immediate reward choice) affects the dependent variable (i.e, immediate reward choice) when the other variables are held constant (Baayen, 2004). For instance, the unique contribution of amount to immediate reward choice is estimated, controlling for the contribution of delay, and vice versa.

To examine the mechanisms underlying individual differences in TD, we extracted three so-called Best Linear Unbiased Predictions (BLUPs; the model's best estimate for each participant's intercept and regression coefficients) for each participant: 1) Average impatience (random intercept) reflects each participant's likelihood to choose the immediate reward for an average amount and delay. Higher values indicate a greater tendency to choose the immediate reward. This BLUP is similar to the frequently used AUC and $k$ rate in that it includes both the influence of delay and amount on choice. Consistent with this point, participants' random intercept was highly correlated with both their AUC (rho=-.93, $p<.001$ for money and snacks) and $k$ rate (money: $r h o=.77, p<.001$; snacks: $r h o=.83, p<.001$ ); 2 ) Amount sensitivity (random amount slope) indexes the unique contribution of the immediate reward amount to TD choice. Higher values indicate an increased likelihood of choosing the immediate reward as its amount increases; 3) Delay sensitivity (random delay slope) indexes the unique contribution of delay to the larger reward to TD choice. Higher values

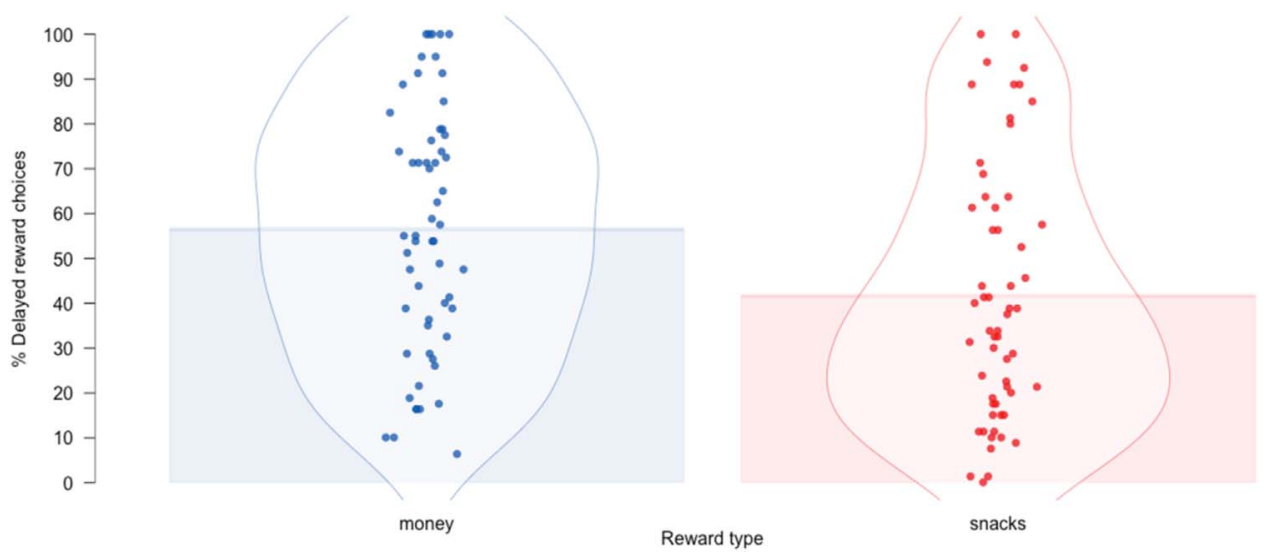

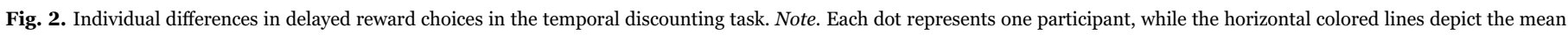

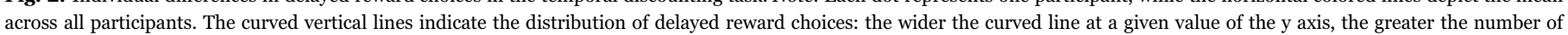
participants who chose this \% of delayed reward choices. 
indicate an increased likelihood of choosing the immediate reward as the delay to the delayed reward increases.

We explored gender differences in the three BLUPs by performing a generalized mixed-effects model analysis that included a fixed intercept and fixed effects for amount of the immediate reward, delay to the delayed reward, gender (sum-to-zero contrast: 1=girl, -1=boy), gender by amount, and gender by delay. Again, for each participant, random adjustments to the fixed intercept and to the slopes of amount, delay, gender, gender by amount, and gender by delay were included. All correlation terms among the random effects were explicitly estimated, and amount and delay were standardized.

Finally, we examined whether the BLUPs were correlated for money and snack choices by performing separate generalized mixed-effects model analyses for the money and snack choices. Both models included a fixed intercept and fixed effects for amount of the immediate reward, and delay. Again, for each participant, random adjustments to the fixed intercept and the slopes of amount and delay were included. All correlation terms among the random effects were explicitly estimated, and amount and delay were standardized.

For the mixed-effects model analyses the optimizer "bobyqa" was used, with a maximum number of $1 \times 10^{9}$ iterations. $P$-values were determined using Likelihood Ratio Tests as implemented in the mixed function of the afex package (Singmann et al., 2015).

We used SPSS version 22.0 to compute Spearman rank-order correlations (rho) of the participant-specific BLUPs with hormone levels, QDQ scale scores, and age. We also computed Spearman rankorder correlations (rho) of the participant-specific BLUPs for money and snack choices, to examine the correlations of the TD components between both reward types.

\section{Analyses of $f M R I$ data}

The fMRI data were preprocessed and analyzed in SPM8 (Wellcome Trust Centre for Neuroimaging, London, UK). From the 30 prescans, optimal weights were calculated for each of the five echo times and used to combine them to one image per volume (Poser et al., 2006). Data were realigned using a rigid body transformation and slice time corrected. The T1-weighted anatomical scan was segmented, and functional images were coregistered to the segmented gray matter image. Finally, data were normalized to an MNI template (ICBM152) and smoothed with a $5 \mathrm{~mm}$ FWHM Gaussian kernel. Three event types were modeled in the general linear model implemented in SPM8: Delayed reward choice, Immediate reward choice, and Control task choice. Events were modeled separately for each block. Events were modeled at the onset of the presentation of the choice (duration=response time of that choice, mean RT=1957 ms), and convolved with a hemodynamic response function and its first-order temporal derivative. Trials in which participants responded faster than $300 \mathrm{~ms}$ or failed to respond within $8000 \mathrm{~ms}$ were not included in the model (to eliminate trials in which participants likely did not process the options sufficiently) (cf. Lansu et al., 2012). Additional regressors were included to model head motion (18 parameters indicating motion relative to the first image of the run: 3 translation and 3 rotation parameters plus the square and first-order derivative of each of these six motion parameters), and to model the objective amount of the immediate reward and delay (parametric regressors, linear trend). To avoid removing low-frequency task-induced effects, we applied a highpass filter with a $320 \mathrm{~s}$ cutoff (the maximum duration of a run).

\section{Whole-brain analyses}

Pairwise contrast images were first computed at the participant level, and subsequently entered in group level one-sample $t$-tests. First, we examined brain regions that were involved in TD choices, independent of individual differences. The following contrasts were computed: TD choice $>$ Control choice, Delayed reward choice $>$ Immediate reward choice, Immediate reward choice $>$ Delayed reward choice (the latter two contrasts could not be computed for 4 participants: 3 participants always chose the delayed reward, while 1 participant always selected the immediate reward). These contrasts were computed combined for the money and snack runs.

Second, we examined how individual differences in TD and hormone levels were associated with brain activity during TD choices. We performed one-sample t-tests on the contrast Delayed reward choice $>$ Immediate reward choice, with either the participant-specific BLUPs or hormone levels as covariates of interest. Specifically, to examine the neural mechanisms of individual differences in TD, three one-sample $t$-tests were run: one with average impatience (random intercept) as a covariate, one with amount sensitivity (random amount slope) as a covariate, and one with delay sensitivity (random delay slope) as a covariate. Two one-sample $t$-tests were run to examine the associations between hormone levels and brain activity: one with estradiol as a covariate, and one with log-transformed testosterone as a covariate. Given the gender-specific nature of pubertal hormones, the hormone analyses were performed for boys and girls separately, with age as an additional covariate.

Third, we compared brain activity during money TD choices versus snack TD choices in two ways. We first examined the neural overlap, that is regions that were active during TD of money and snacks, using a conjunction analysis under the conjunction null hypothesis (Nichols et al., 2005). We examined the neural overlap for money and snacks: 1) during TD choices compared to Control choices (conjunction: TD money choice $>$ Control choice \& TD snack choice $>$ Control choice); 2) during delayed reward choices compared to immediate reward choices (conjunction: money delayed reward choice > money immediate reward choice \& snack delayed reward choice $>$ snack immediate reward choice) and; 3) during immediate reward choices compared to delayed reward choices (conjunction money immediate reward choice $>$ money delayed reward choice \& snack immediate reward choice > snack delayed reward choice).

Second, we directly compared brain activity during TD choices for money versus snacks by examining brain areas that were more active for TD of money than TD of snacks, and vice versa. We computed pairwise contrast images at the participant level, which were subsequently entered in one-sample $t$-tests at the group level. The difference in mean reward valuation ratings of the money and snack rewards were included as a covariate, to account for differences in valuation between them. We compared activity during TD choices for money versus snacks, independent of whether participants chose the immediate or delayed reward (contrasts: TD choice money $>$ TD choice snacks, TD choice snacks $>$ TD choice money). We further compared activity during delayed reward choices for money versus snacks (contrasts: Delayed reward choice money $>$ Delayed reward choice snacks, Delayed reward choice snacks $>$ Delayed reward choice money), and during immediate reward choices for money versus snacks (contrasts: Immediate reward choice money $>$ Immediate reward choice snacks, Immediate reward choice snacks $>$ Immediate reward choice money).

All whole-brain analyses were corrected for multiple comparisons using FWE- correction $(p<.05$ at the cluster level, with a clusterforming threshold of $p<.001$ ).

\section{Region of interest (ROI) analyses}

In order to further investigate the neural mechanisms underlying individual differences in TD, we selected ROIs based on prior fMRI studies that focused on TD in adolescents or conceptually similar constructs (e.g., anticipating delays and return sensitivity). We used MarsBar 0.43 (Brett et al., 2002) to extract parameter estimates centered on peak voxels (spheres with a 6-mm radius) reported in these studies. We extracted parameter estimates for the contrast Delayed reward choice $>$ Immediate reward choice. Note that this contrast was unbiased in that it probes brain activity during TD choices, independent of the individual differences in TD and hormone levels with which we subsequently correlated the brain activity (see 
below). The MNI coordinates of the ROIs and the studies from which they were derived are reported in Table S2.

The right and left VS were selected to study associations with average impatience (random intercept), amount sensitivity (random amount slope) and hormones. The right and left vmPFC were chosen to examine associations with average impatience (random intercept), and amount sensitivity (random amount slope). The right DLPFC and superior parietal cortex were selected for associations with average impatience (random intercept). The right amygdala and insula were used to study associations with delay sensitivity (random delay slope).

Spearman rank-order correlations examined associations between activation in these ROIs and average impatience, amount sensitivity, and delay sensitivity. We computed partial correlations, controlling for age, to examine the associations of testosterone and estradiol levels with ROI activation and TD. Due to the sex-specific nature of these hormones, these analyses were performed separately for boys and girls.

A false discovery rate (FDR) correction was applied to control for multiple testing (see Benjamini and Hochberg (1995), for the procedure of computing an FDR-corrected $p$-value), resulting in a significance threshold of $p<.013$.

\section{Results}

\section{Behavioral results}

\section{Correlations}

We examined correlations between the three participants-specific BLUPs, and whether they were associated with self-reported daily life discounting and delay aversion (QDQ; to examine their ecological validity) and age. All correlations are presented in Table 1 , while we focus here on those correlations surviving FDR-correction for multiple comparisons. Self-reported delay discounting in daily life (QDQ) was positively correlated with average impatience, indicating that participants who were highly impatient in the TD task also reported increased discounting of delayed rewards in daily life. The participant-specific BLUPs were all highly correlated with each other, but in opposite directions. As expected, average impatience and delay sensitivity were positively correlated, indicating that participants who frequently selected the immediate reward, selected it particularly frequently as the delay preceding the delayed reward increased. Unexpectedly, amount sensitivity was negatively correlated with both average impatience (see Fig. 3) and delay sensitivity, indicating that participants who rarely selected the immediate reward, selected it when its magnitude was high. This is shown in Fig. S4, in which the number of immediate reward choices is plotted as a function of reward magnitude for the participant with the highest amount sensitivity score in this study. As can be seen in Fig. S4, this participant only selected the immediate reward in $10 \%$ of trials, but when she did select it, it was always when its magnitude was highest (8 Euros). Together, these

Table 1

Correlations (rho) between the study variables.

\begin{tabular}{lllllll}
\hline & 1. & 2. & 3. & 4. & 5. & 6. \\
\hline 1. Average impatience & - & & & & & \\
2. Amount sensitivity &.$- .67^{* *}$ & - & & & & \\
3. Delay sensitivity & $\mathbf{. 8 7}^{* *}$ & $-.50 *$ & - & & & \\
4. QDQ - DD & $\mathbf{. 3 4}$ & $-.27^{*}$ & .22 & - & & \\
5. QDQ- DA & $.23^{+}$ & -.10 & .06 & .20 & - & \\
6. Age & -.12 & $.25^{+}$ & -.03 & $-.24^{+}$ & -.18 & - \\
\hline
\end{tabular}

Note. QDQ-DD=Quick Delay Questionnaire- Delay Discounting scale, QDQ-DA=Quick Delay Questionnaire- Delay Aversion scale. Only correlations printed in bold survived FDR-correction for multiple comparisons.

*** $p<.001$.

${ }^{*} p<.05$.

$+p<.10$. correlations suggest that the TD choices of adolescents who are relatively impatient are driven by a strong sensitivity to delay preceding the larger reward, and a relative insensitivity to amount of the immediate reward. In contrast, TD choices of adolescents who are relatively patient seem to be driven by a strong sensitivity to amount and a relative insensitivity to delay.

We examined whether the three participant-specific BLUPs were correlated between money and snack choices. There were strong positive correlations between money and snacks for average impatience ( $r h o=.78, p<.001)$, delay sensitivity ( $r h o=.71, p<.001)$, and amount sensitivity (rho=.55, $p<.001)$.

\section{Gender differences}

There was a main effect of gender on TD choice $(B=2.36, S E=0.98$, $\chi^{2}(1)=5.69, p=.02$ ), indicating that girls were more impatient than boys. The gender by delay sensitivity interaction was significant, indicating that girls were more sensitive to delays than boys $(B=2.07$, $\left.S E=0.96, \chi^{2}(1)=9.92, p=.002\right)$. Gender and amount sensitivity did not show a significant interaction $\left(B=-0.15, S E=0.12, \chi^{2}(1)=1.42, p=.23\right)$.

\section{Mixed-effects model analysis of TD task choices}

The delay preceding the delayed reward, the amount of the immediate reward, reward type (snacks vs. money), and the reward type by delay interaction all significantly influenced participants' TD choices. Participants were more likely to choose the immediate reward as the delay to the delayed reward increased $\left(B=6.36, S E=1.06, \chi^{2}(1)\right.$ $=27.34, p<.001$ ), and as the amount of the immediate reward increased $\left(B=1.54, S E=0.15, \chi^{2}(1)=60.89, p<.001\right)$. Participants showed a stronger preference for immediate rewards when making choices involving snacks than money $\left(B=1.58, S E=0.30, \chi^{2}(1)=22.97\right.$, $p<.001)$. There was a significant interaction between reward type and delay $\left(B=0.79, S E=0.33, \chi^{2}(1)=5.89, p=.02\right)$ (see Fig. 4), indicating that delay contributed more strongly to TD choice for snack rewards than money. Reward type did not significantly interact with amount $\left(B=-0.07, S E=0.08, \chi^{2}(1)=0.72, p=.39\right)$.

\section{fMRI results}

\section{Neural regions involved in TD choices}

Consistent with prior TD studies (see Scheres et al. (2013), for a review), a frontoparietal network was more strongly activated during TD choices than control task choices. This network included the angular gyrus, mOFC, DLPFC, VLPFC, dACC, and anterior PFC (see Table S3 and Fig. 5).

Delayed reward choices, compared to immediate reward choices, activated the superior parietal cortex (see Table S4). There were no regions more active for immediate reward choices than for delayed reward choices.

There were no significant age or gender differences (all $p$ 's $>.22$ ) in brain activity during TD choices for any contrast (TD choice $>$ Control choice, Delayed reward choice $>$ Immediate reward choice, and Immediate reward choice $>$ Delayed reward choice).

\section{Individual differences}

Whole-brain analyses. Average impatience was positively associated with activation of frontoparietal regions (lateral PFC, inferior parietal cortex and dACC) during delayed reward choices, compared to immediate reward choices (see Table S5 and Fig. 6A). This means that participants who frequently chose the immediate reward, activated frontoparietal brain areas more during delayed reward choices than during immediate reward choices.

Amount sensitivity was positively correlated with dACC activity during immediate reward choices, compared to delayed reward choices 


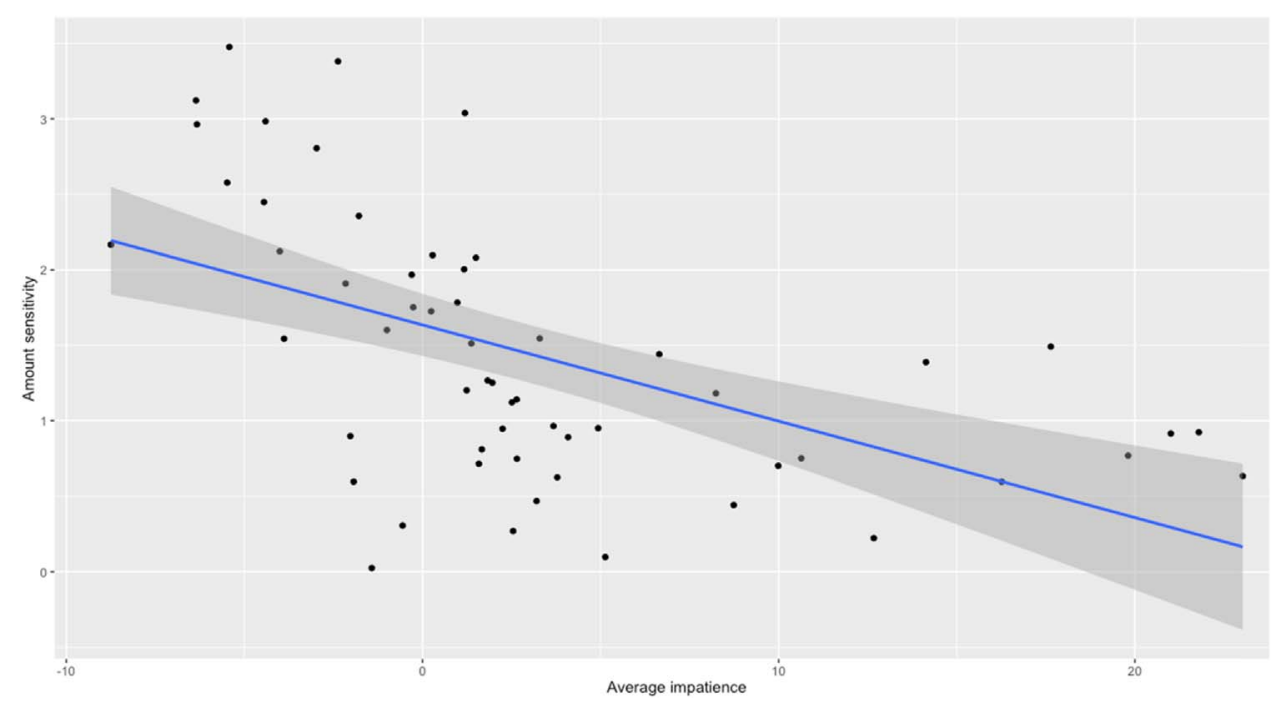

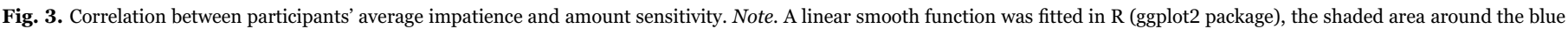

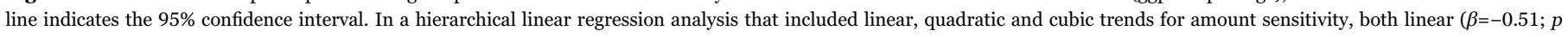
$<.001)$ and cubic $(\beta=0.76 ; p=.009)$ associations were significant.

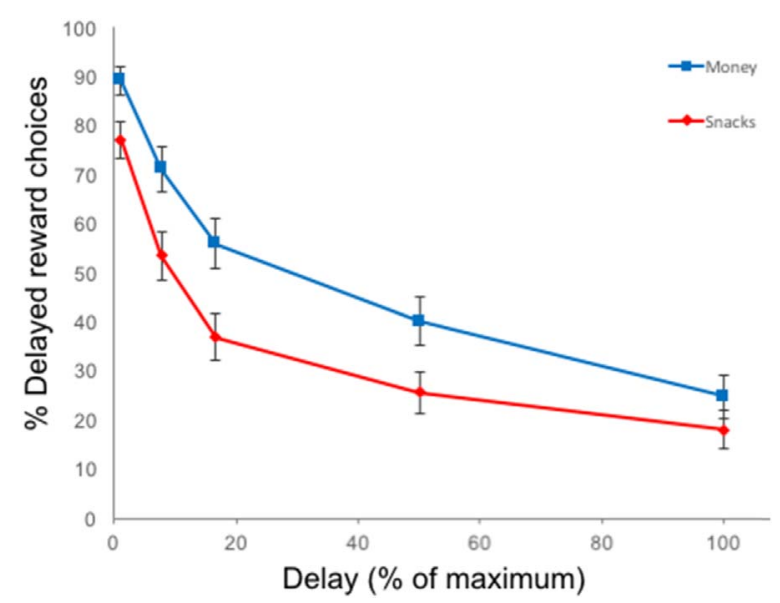

Fig. 4. Interaction between reward type and delay showing greater temporal discounting of snacks. Error bars represent the standard error of the mean.

(see Table S5 and Fig. 6B). This means that participants who chose the immediate reward more often as its amount increased activated the dACC more during immediate reward choices than during delayed reward choices.

Delay sensitivity was positively correlated with activation of the parietal cortex during delayed reward choices, compared to immediate reward choices (see Table S5 and Fig. 6C). This means that participants who selected the immediate reward more frequently as the delay preceding the larger reward increased, activated the parietal cortex more during delayed reward choices than immediate reward choices.

ROI analyses. Average impatience was positively correlated with activity of the left VS ( $r h o=.38, p=.004)$, right VS ( $r h o=.40, p=.003)$, and DLPFC (rho=.46, $p<.001)$ during delayed reward choices (contrast: delayed reward choice $>$ immediate reward choice) (see Fig. 7A). Average impatience was also positively correlated with activation of the left vmPFC ( $r h o=-.40, p=.003$ ) during immediate reward choices (contrast: immediate reward choice $>$ delayed reward choice). Amount sensitivity was positively correlated with activation of the left VS (rho=-.40, $p=.003)$ and right VS $(r h o=-.37, p=.005)$ during immediate reward choices (contrast: immediate reward choice > delayed reward choice) (see Fig. 7B). Delay sensitivity was not correlated with activity in the selected ROIs.
For the contrast TD choice $>$ Control choice, there were no significant correlations that survived correction for multiple comparisons.

Controlling for an unequal distribution of choices across participants. Given that participants showed great variability in their TD choices, the power to detect brain activity associated with delayed vs. immediate reward choices may have differed. For instance, participants who made an approximately equal number of immediate and delayed reward choices would have equal power to detect brain activity associated with both choices. However, participants with a strong preference for either delayed or immediate rewards would have greater power to detect brain activity associated with their preferred choice. We explored whether this influenced our findings by creating a variable that indicated the extent to which a participant's choices deviated from an equal distribution of immediate and delayed reward choices. Specifically, we computed how much each participant's choices deviated from 80 choices (i.e., $50 \%$ of the 160 TD choices we administered), so that lower scores indicated an equal distribution of choices (e.g., someone who made 80 immediate reward choices and 80 delayed reward choices would receive a score of 0 ), while higher scores indicated an unequal distribution of choices and thus relatively less power to detect brain activity for either delayed or immediate reward choices (e.g., someone who made 30 immediate reward choices and 130 delayed reward choices would receive a score of 50, as would someone who made 130 immediate and 30 delayed reward choices). We included this variable as a covariate in all the above mentioned whole-brain and ROI analyses that focused on individual differences in TD.

All of the whole-brain and ROI findings remained significant at a corrected threshold. This suggests that our findings were not driven by differences in the power to detect brain activity between participants.

\section{Comparison of TD of money and snacks}

First, we performed conjunction analyses to examine brain areas that showed overlap for money and snack TD choices, independent of whether the delayed or immediate reward was selected. TD choices for money and snacks (compared to control task choices) both activated areas in the parietal cortex (see Table S6 and Fig. 8). When we examined brain areas that showed overlap for money and snack TD choices during delayed reward choices or immediate reward choices 


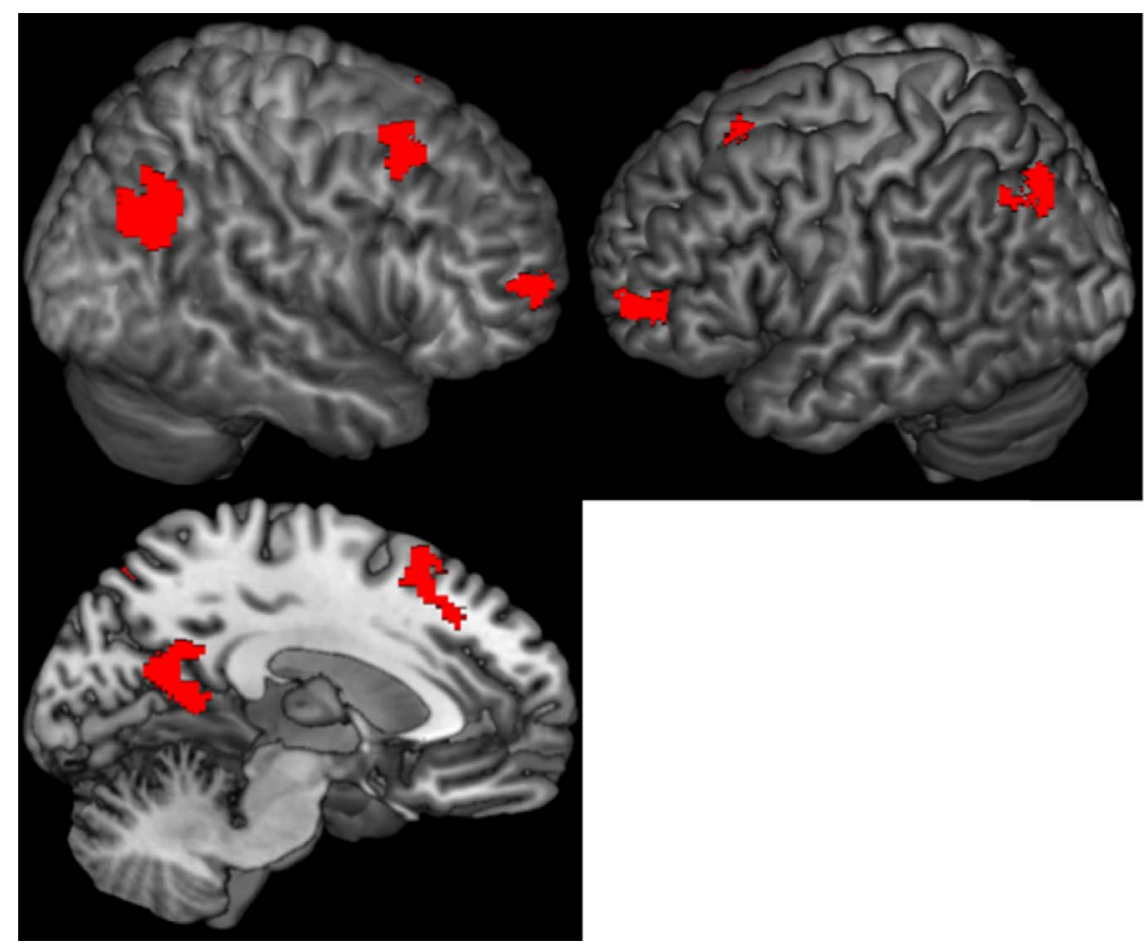

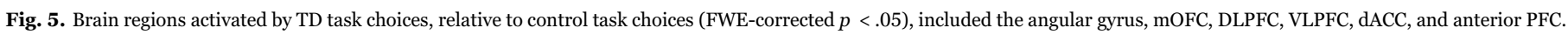
See Table S3 for details of specific activated regions.

specifically, no overlapping regions were found.

Second, we directly compared brain activity during TD choices for money and snacks. No regions were more active for one reward type or the other. To control for Type II error, we also compared activity during choices for money and snacks using a more lenient threshold $(p<.001$, uncorrected), but there were no significant differences then either.

\section{Hormone analyses}

Testosterone and estradiol levels were not significantly associated with average impatience, or amount or delay sensitivity in boys or girls (all $p$ 's > .19). Hormone levels also were not correlated with brain activity during TD choices, neither at the whole-brain level nor the ROI level. Hormone levels also were not significantly correlated with brain activity when we used a more lenient threshold ( $p<.001$, uncorrected).

\section{Discussion}

This study examined in adolescents: 1) the neural mechanisms underlying individual differences in TD; 2) overlap and differences between TD of money and snacks; 3 ) the association between testosterone and estradiol levels and (neural activation during) TD choices. In order to study individual differences, we used a mixed-effects model approach to determine participants' average impatience, and to further decompose TD choices into two components: 1) amount sensitivity; and 2) delay sensitivity. This approach allowed us to delve into the distinct factors underlying the neural mechanisms responsible for individual differences in TD choices.

In order to support our interpretation of the activation of brain areas that correlated significantly with TD, we used Neurosynth (Yarkoni et al., 2011) to determine the posterior probabilities (pp) of the peak voxel of each brain area. The posterior probabilities reflect the conditional probability of a term being used in a study, conditional on activation being present at this voxel. For example, $\mathrm{pp}=0.80$ for 'reward' at a voxel would mean that $80 \%$ of studies that report activation at that voxel also use the term 'reward' in their abstracts.

\section{Individual differences in $T D$}

In line with our hypotheses, we found that individual differences in TD were associated with differential activation of frontoparietal brain areas, the VS and vmPFC during TD choices. Consistent with prior research, adolescents who were on average highly impatient engaged the lateral $\mathrm{PFC}$ ( $\mathrm{pp}=0.88$ for 'cognitive performance'), inferior parietal lobule ( $\mathrm{pp}=0.75$ for 'executive functions') and $\mathrm{dACC}$ ( $\mathrm{pp}=0.83$ for 'conflicting') more strongly during delayed reward choices compared to immediate reward choices (Stanger et al., 2013). These frontoparietal regions are involved in cognitive control, conflict monitoring and attention (Bunge and Wright, 2007). It is plausible that more impulsive adolescents recruited these areas preferentially during delayed reward choices because these choices require more effortful control to delay gratification, consistent with the finding that highly impulsive adolescents were also highly sensitive to delay. Delayed reward choices might have been more effortful for highly impulsive adolescents, because they may show decreased neural efficiency and frontoparietal maturity, relative to their less impulsive peers. Consistent with this interpretation, a recent longitudinal study in adolescents reported decreased VLPFC activation over time, and those adolescents who showed the strongest reductions also showed the largest declines in risk-taking $(\mathrm{Qu}$ et al., 2015).

In addition, participants who were on average highly impatient in the TD task also engaged the $\mathrm{vmPFC}$ ( $\mathrm{pp}=0.75$ for 'reward') more strongly during immediate reward choices and the VS ( $\mathrm{pp}=0.65$ for 'regulating') more strongly during delayed reward choices, consistent with prior studies (Hariri et al., 2006; Stanger et al., 2013). While the finding that more impatient adolescents exhibited greater VS activity during delayed reward choices is in line with a recent study (Stanger et al., 2013), it does not fit with the notion that the VS is uniquely sensitive to the immediacy of rewards (McClure et al., 2004). Nevertheless, the VS has multiple functions, and has been shown to play an important role in the regulation of emotions and of impulsive behavior in adolescents and adults as well (Hare et al., 2005; Masten et al., 2009; Pfeifer et al., 2011). Highly impatient adolescents were also highly sensitive to delay, as indicated by the strong positive 
A

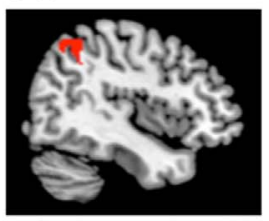

inferior parietal lobule

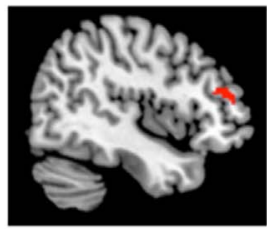

inferior frontal gyrus

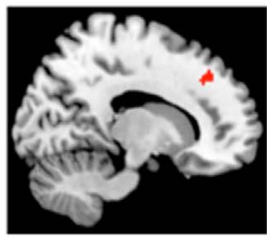

dACC

B

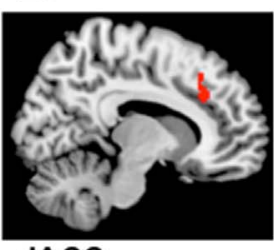

dACC

C

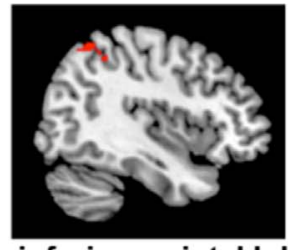

inferior parietal lobule

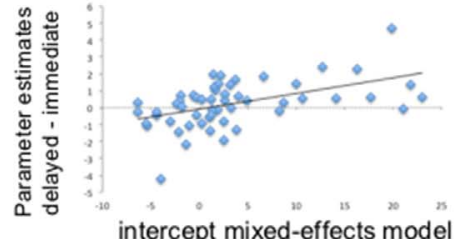

intercept mixed-effects model
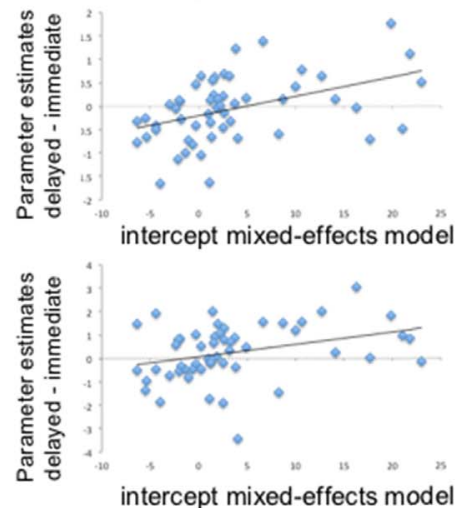

intercept mixed-effects model
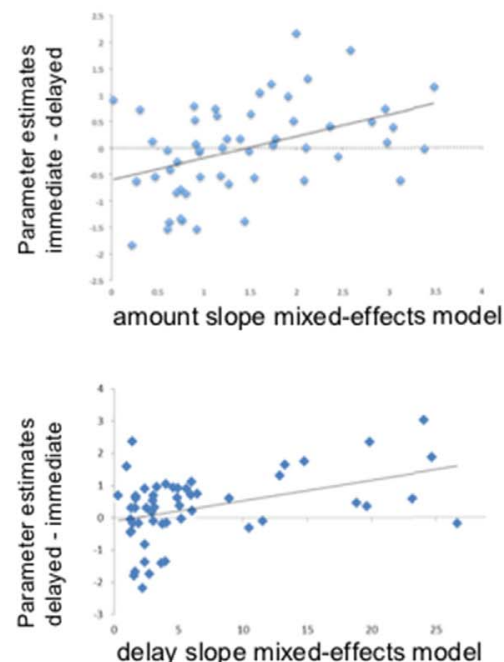

delay slope mixed-effects model

Fig. 6. Associations between brain activity, identified by whole-brain analyses (FWEcorrected $p<.05$ ), and A) average impatience; B) amount sensitivity; C) delay sensitivity.

correlation between average impatience and delay sensitivity in this study. Thus, it may be speculated that the increased VS activity during delayed reward choices might reflect the fact that more impatient adolescents needed to regulate their emotions more strongly than their less impatient peers when they made these choices.

In line with our hypotheses, participants who were more sensitive to immediate reward amount demonstrated more VS activity during immediate reward choices. Unexpectedly, adolescents who were more sensitive to immediate reward amount also engaged the dACC ( $\mathrm{pp}=0.73$ for 'salience network') more during immediate reward choices. This region has been shown to encode for increasing subjective value of rewards in adults (Kable and Glimcher, 2010). Increased activity might reflect the emotional salience of immediate rewards for adolescents who are more sensitive to immediate reward amount (Liu et al., 2011).

Adolescents who were more sensitive to delay in the TD task activated the inferior parietal cortex ( $\mathrm{pp}=0.71$ for 'response inhibition'; $\mathrm{pp}=0.69$ for 'attentional control') more during delayed reward choices. This region has been implicated in cognitive control functions, such as attention and inhibition (Cavanna and Trimble, 2006). Adolescents

who are more sensitive to delay may show increased inferior parietal lobule activation during delayed reward choices, because they require more effortful control during these choices. Contrary to our hypotheses, delay sensitivity was not associated with limbic brain areas such as the amygdala and insula. In prior research, enhanced amygdala and insula responses to anticipated delays were observed in individuals with ADHD (Lemiere et al., 2012; Plichta et al., 2009; Wilbertz et al., 2013). This discrepancy with our findings might be because these responses are specific to individuals with ADHD, or to tasks in which all delays are actually experienced (experiential TD tasks). Experiential tasks are arguably highly emotionally salient (Scheres et al., 2014), and might tap into an affective component of delay sensitivity (i.e., the subjective feeling of distress induced by the experience of a delay). The TD task we used may have tapped into a more cognitive component of delay sensitivity (i.e., imagining how one would feel if one would experience a delay), since delay sensitivity as assessed with the TD task was not correlated with self-reported delay aversion in daily life (i.e., negative feelings that are experienced during imposed delays). Future studies should test this possibility by using experiential TD tasks.

Collectively, our decomposition of TD choices into its underlying components has also provided unique and novel insights into the underlying mechanisms of individual differences in TD in adolescents. Previous studies on individual differences in TD (Benningfield et al., 2014; de Water et al., 2014; Ripke et al., 2012; Stanger et al., 2013) have used outcome measures that reflect the average preference for immediate rewards, such as the area under the curve (AUC; Myerson et al., 2001) and $k$ rate (Mazur et al., 1987). These measures do not enable the investigation of the mechanisms underlying steep TD (van den Bos and McClure, 2013), such as sensitivity to the immediate reward amount and to the delay preceding the larger reward. Decomposing TD choices into its underlying components could aid the development of more personalized interventions to reduce steep TD, as adolescents who are driven by delay sensitivity could benefit from different interventions than those who are driven by amount sensitivity. At the behavioral level, we showed that both amount sensitivity and delay sensitivity contributed to adolescents' average impatience, but in opposite directions. That is, adolescents who are on average highly impatient are particularly driven by a heightened delay sensitivity and less by their amount sensitivity. Conversely, adolescents who are on average highly patient seem to be primarily driven by a heightened immediate reward amount sensitivity (i.e., they only select this reward when its magnitude is high) and less by sensitivity to delays. Highly patient adolescents may strongly prefer the delayed reward because it is higher in magnitude than the immediate rewards. Future studies should test this hypothesis by systematically varying the magnitude of the delayed reward as well. Further, at the neural level, we showed that distinct neural mechanisms are associated with distinct underlying components of TD choice. Consistent with prior studies, we demonstrated that both frontoparietal and limbic brain areas were hyperactive during TD choices in adolescents who were on average highly impatient. In addition, we showed that activity of the parietal cortex was uniquely associated with delay sensitivity, while activity of the VS and dACC was uniquely associated with amount sensitivity.

Our findings on the neural mechanisms underlying individual differences in TD in typically developing adolescents could serve as a template for aberrant neural functioning in adolescents with impulsecontrol disorders (e.g., ADHD, substance abuse, gambling), and may inspire interventions to reduce impulsivity. While steep TD has been observed across different impulse-control disorders (Bickel et al., 2012), the mixed-effects model approach we used may reveal subtle differences between adolescents with different impulse-control disorders, as they may show differences in particular components of TD choice (i.e., amount sensitivity and delay sensitivity) (cf. Figner et al., 2010; Foerde et al., 2016). If adolescents with different impulse-control disorders would indeed show differences in specific components of TD choice, this could inspire hypotheses about the specific neural mechan- 


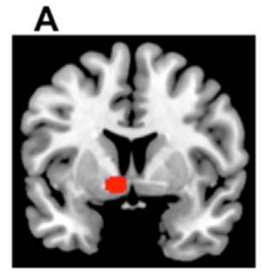

left VS

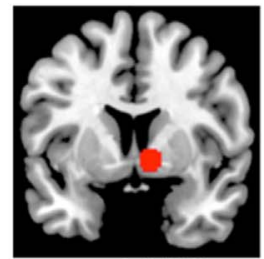

right VS

B

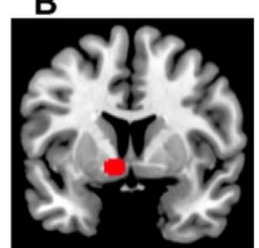

left VS

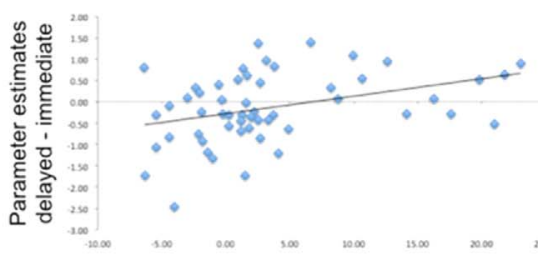

intercept mixed-effects model
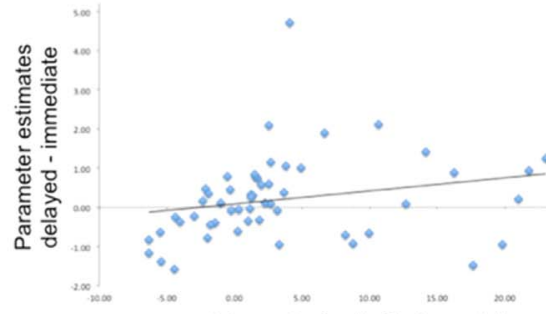

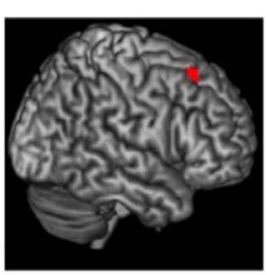

DLPFC

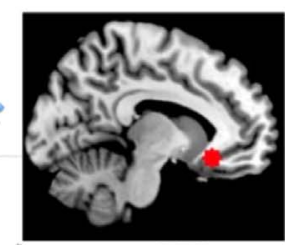

vmPFC

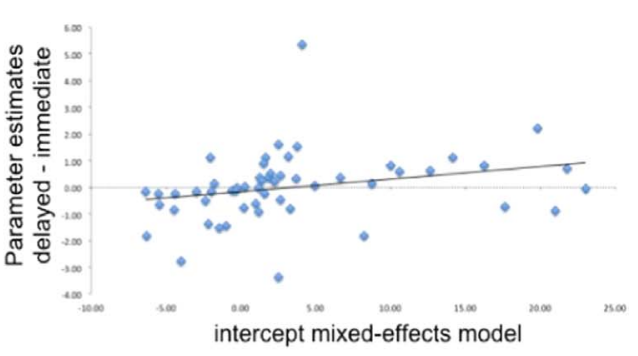

intercept mixed-effects model

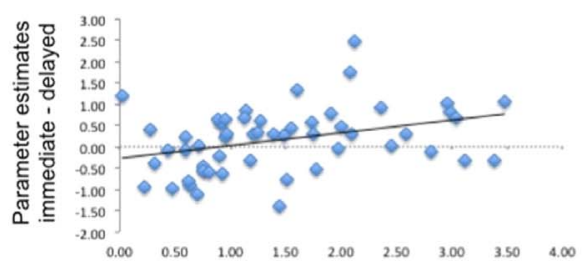

amount slope mixed-effects mode

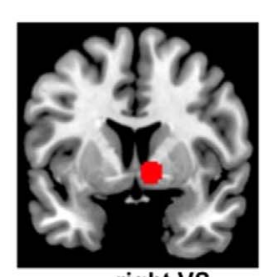

right VS
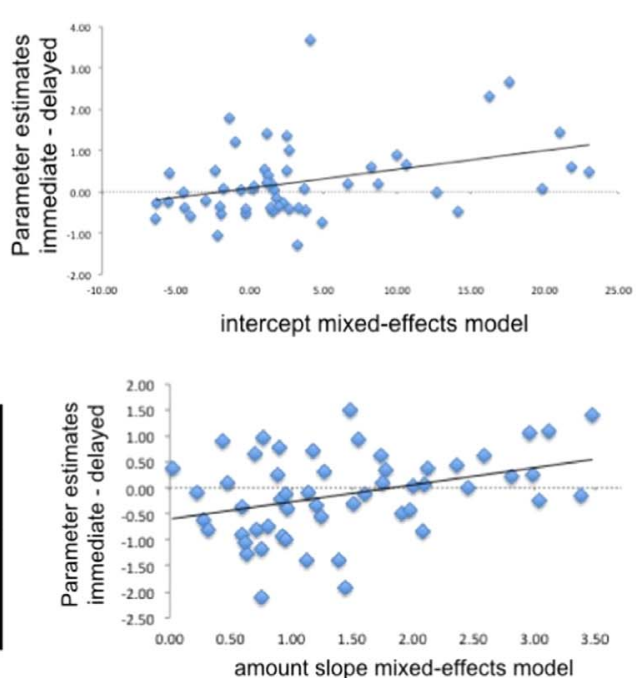

Fig. 7. Associations between brain activity in a priori defined regions of interest and: A) average impatience; B) amount sensitivity.

isms contributing to steep TD in different disorders, as we showed that amount and delay sensitivity have different neural correlates.

We showed that highly impatient adolescents are particularly driven by a heightened sensitivity to delays, suggesting that interventions targeting delay tolerance may be particularly effective (Neef et al., 2001). We have further identified frontoparietal and limbic (i.e., VS) brain areas that are associated with steep discounting of delayed rewards; activation of these brain areas could be altered by recently developed neurofeedback training programs, such as real time fMRI neurofeedback (Cohen Kadosh et al., 2016). Further, we showed that differential recruitment of the lateral and medial PFC is related to steep $\mathrm{TD}$, and several cognitive interventions have been shown to alter activation of these regions and reduce TD in young adults, including working memory training (Bickel et al., 2011; Wesley and Bickel, 2014) and episodic prospection training (Benoit et al., 2011; Peters and Buechel, 2010).

\section{Comparison of TD of money and snack rewards}

Consistent with our hypotheses and prior studies (Estle et al., 2007; Jimura et al., 2011), snack rewards were discounted more steeply than monetary rewards. Estle and colleagues (2007) showed that young adults discounted alcohol just as steeply as candy rewards, and both rewards were discounted more steeply than money. TD of primary rewards may therefore better predict alcohol use than TD of money (Jimura et al., 2011). Future studies need to examine this hypothesis in adolescents who are at-risk for developing alcohol abuse problems. Despite the behavioral difference between TD of money and snack rewards, there were no brain areas more active for one reward type vs. the other. This might be due to the fact that TD of money and snacks were highly correlated, more highly than in adult studies (Jimura et al., 2011), possibly because many adolescents use the money they earn or receive from their parents to buy snacks (anecdotally, several partici-
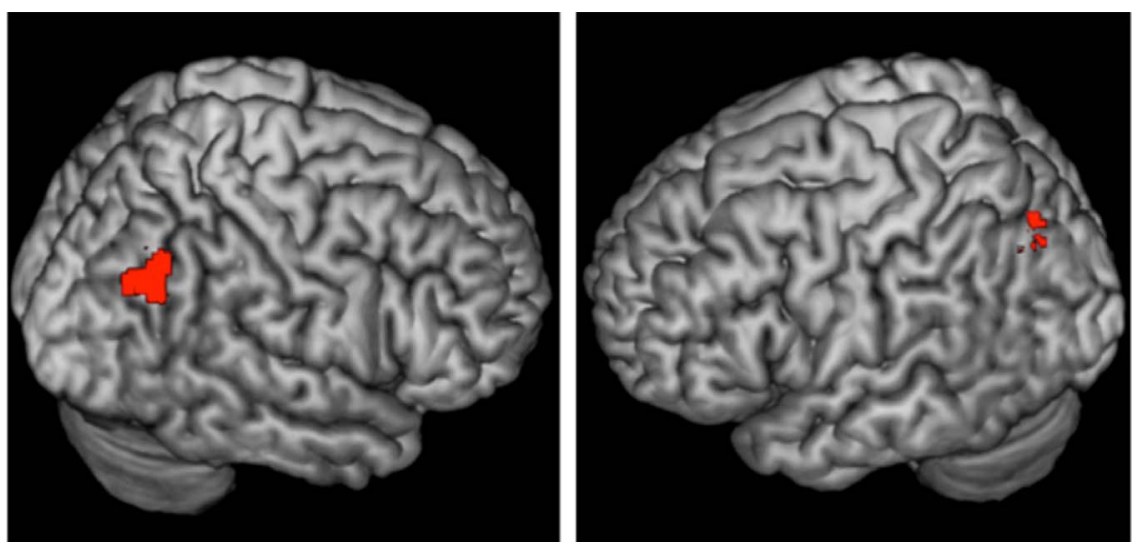

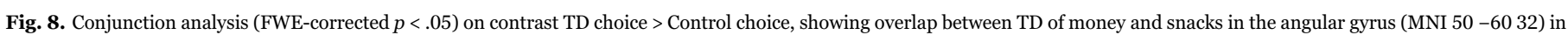
the left panel, and the inferior parietal lobule (MNI -34 -66 40) in the right panel. 
pants indicated that they used their money to buy snacks). Prior studies also reported highly overlapping neural substrates for delayed monetary and primary reward choices (Hare et al., 2009, 2014; McClure et al., 2004, 2007). Consistent with McClure et al.'s findings (2004, 2007), choices for both reward types showed neural overlap in the parietal cortex in the present study. Given the role of the parietal cortex in cognitive control, the overlap between TD of money and snacks in this region may reflect that exerting effortful control to guide decisions involving rewards and delays is important for TD choices in general, independent of the type of reward.

\section{Pubertal hormones}

Testosterone and estradiol levels were surprisingly neither associated with TD choices, nor with neural activity during these choices. Previous behavioral studies, however, also did not find statistically significant associations between testosterone levels and TD (Bromberg et al., 2015; Peper et al., 2013), although Bromberg et al. found a nonsignificant trend towards a positive association between testosterone and a preference for immediate rewards in adolescent boys. Two previous neuroimaging studies showed that testosterone levels were positively correlated with VS responses to reward processing (Braams et al., 2015; Op de Macks et al., 2011). In contrast with the present study, these investigations employed gambling tasks in which participants received or lost real rewards. It has been proposed that pubertal hormones particularly influence brain areas involved in motivational or emotional tendencies towards sensation-seeking behavior (e.g., the VS) (Crone and Dahl, 2012). Thus, it might be that associations between hormone levels and brain activation will only be observed when emotionally salient tasks that draw on sensation-seeking tendencies are used, such as gambling tasks involving real money, that include elements of uncertainty and surprise. The TD task of this study and most prior studies might be less emotionally salient than such gambling tasks, since participants do not experience all rewards and delays. Future studies should examine whether associations between hormones and brain activity are observed in experiential TD tasks, in which all rewards and delays are experienced, making these tasks more emotionally salient than the TD task of the present study. Additionally, the relatively modest sample size for the pubertal hormone analyses may have provided insufficient statistical power to detect associations between hormone levels and neural activity underlying TD.

\section{Limitations and future directions}

This study had some limitations. Some participants could not be included in analyses, because they showed no variability in their TD choices. This issue could be reduced by using designs in which the amount of the immediate reward is adjusted based on participants' choices (Christakou et al., 2011), or in which participant-specific choice sets are presented based on a pre-test outside of the scanner (van den Bos et al., 2015). Further, we used a potentially real TD task, in which one choice was randomly selected and paid to participants at the corresponding delay, under the assumption that participants would choose as if every choice might have real-life consequences. The effects of amount sensitivity and delay sensitivity on TD choices could have been even stronger if all rewards and delays were experienced, such as in experiential or "real" TD tasks (cf. Scheres et al., 2013, 2006).

Future studies could extend the current study in several ways. First, longitudinal studies could examine how changes in the underlying components of TD choices are associated with changes in brain activity, they could elucidate whether functional maturation of frontoparietal areas is delayed in adolescents who are more impatient, and whether brain activation at one time predicts impatience at a later time. Second, although the current study could serve as a template for neural mechanisms of components of impulsivity in adolescents with impulse-control problems (e.g., ADHD and substance abuse), such participants need to be included in future studies to directly compare their brain activity to typically developing adolescents.

\section{Conclusions}

In summary, individual differences in TD were associated with differential activity of brain areas implicated in cognitive control (lateral PFC and parietal cortex) and reward valuation (VS and vmPFC) during TD choices. We used mixed-effects modeling to study adolescents' average impatience, and to decompose their TD choices into amount sensitivity and delay sensitivity. Different underlying components contributed to two extremes of the continuum of impulsive choice: highly impatient adolescents were particularly sensitive to delay, while highly patient adolescents were particularly influenced by amount sensitivity. Distinct brain areas were associated with distinct components of TD choice. Both cognitive control and reward valuation areas were associated with average impatience, while reward valuation areas were uniquely implicated in amount sensitivity, and cognitive control areas were uniquely involved in delay sensitivity. Snack rewards were discounted more steeply than money, and the neural correlates of TD for both money and snacks overlapped in the parietal cortex. Pubertal hormones were not robustly correlated with TD choices or neural activity. These findings underscore the value of using models designed to disentangle how underlying components of impulsive choice contribute to a seemingly identical behavioral outcome, and should be extended to adolescents with impulse-control problems.

\section{Acknowledgements}

This study was funded by the Behavioural Science Institute at Radboud University, Nijmegen, the Netherlands. We would like to thank all participants and their parents. We are grateful to Ili Ma for providing the Matlab script to combine the multiple echoes. We thank Kim Fairley for sharing a Matlab script to add parametric regressors in SPM, and Alan Sanfey and his team members for their helpful feedback on the design and analyses of this study. We are indebted to Jana Kruppa for assistance during scanning, and Paul Gaalman for technical assistance. We thank Hans van Pelt for his suggestions regarding optimal collection of the saliva samples that were used to determine hormone levels. Finally, we thank Isabel Woyke for providing the $\mathrm{R}$ script to compute subjective amount and delay values.

\section{Appendix A. Supplementary material}

Supplementary data associated with this article can be found in the online version at doi:10.1016/j.neuroimage.2017.04.013.

\section{References}

Achenbach, T.M., Rescorla, L.A., 2001. Manual for the ASEBA School-Age Forms \& Profiles. University of Vermont, Research Center for Children, Youth, \& Families, Burlington, Vermont.

Audrain-McGovern, J., Rodriguez, D., Epstein, L.H., Cuevas, J., Rodgers, K., Wileyto, E.P., 2009. Does delay discounting play an etiological role in smoking or is it a consequence of smoking? Drug Alcohol Depend. 103 (3), 99-106. http://dx.doi.org/ 10.1016/j.drugalcdep.2008.12.019.

Baayen, R.H., Davidson, D.J., Bates, D.M., 2008. Mixed-effects modeling with crossed random effects for subjects and items. J. Mem. Lang. 59 (4), 390-412. http:// dx.doi.org/10.1016/j.jml.2007.12.005.

Ballard, K., Knutson, B., 2009. Dissociable neural representations of future reward magnitude and delay during temporal discounting. Neuroimage 45 (1), 143-150. http://dx.doi.org/10.1016/j.neuroimage.2008.11.004.

Barr, D.J., Levy, R., Scheepers, C., Tily, H.J., 2013. Random effects structure for confirmatory hypothesis testing: keep it maximal. J. Mem. Lang. 68 (3), 255-278. http://dx.doi.org/10.1016/j.jml.2012.11.001.

Bates, D., Maechler, M., Bolker, B., Walker, S., 2015. Fitting linear mixed-effects models using lme4. J. Stat. Softw. 67 (1), 1-48. http://dx.doi.org/10.18637/jss.v067.i01.

Benjamini, Y., Hochberg, Y., 1995. Controlling the false discovery rate- a practical and powerful approach to multiple testing. J. R. Stat. Soc. Ser. B-Methodol. 57 (1), 289-300.

Benningfield, M.M., Blackford, J.U., Ellsworth, M.E., Samanez-Larkin, G.R., Martin, 
P.R., Cowan, R.L., Zald, D.H., 2014. Caudate responses to reward anticipation associated with delay discounting behavior in healthy youth. Dev. Cogn. Neurosci. 7, 43-52. http://dx.doi.org/10.1016/j.dcn.2013.10.009.

Benoit, R.G., Gilbert, S.J., Burgess, P.W., 2011. A neural mechanism mediating the impact of episodic prospection on farsighted decisions. J. Neurosci. 31 (18), 6771-6779. http://dx.doi.org/10.1523/jneurosci.6559-10.2011.

Bickel, W.K., Jarmolowicz, D.P., Mueller, E.T., Koffarnus, M.N., Gatchalian, K.M., 2012. Excessive discounting of delayed reinforcers as a trans-disease process contributing to addiction and other disease-related vulnerabilities: emerging evidence. Pharmacol. Ther. 134 (3), 287-297. http://dx.doi.org/10.1016/ j.pharmthera.2012.02.004

Bickel, W.K., Pitcock, J.A., Yi, R., Angtuaco, E.J.C., 2009. Congruence of BOLD response across intertemporal choice conditions: fictive and real money gains and losses. J. Neurosci. 29 (27), 8839-8846. http://dx.doi.org/10.1523/jneurosci.5319-08.2009.

Bickel, W.K., Yi, R., Landes, R.D., Hill, P.F., Baxter, C., 2011. Remember the future: working memory training decreases delay discounting among stimulant addicts. Biol. Psychiatry 69 (3), 260-265. http://dx.doi.org/10.1016/j.biopsych.2010.08.017.

Braams, B.R., van Duijvenvoorde, A.C.K., Peper, J.S., Crone, E.A., 2015. Longitudinal changes in adolescent risk-taking: a comprehensive study of neural responses to rewards, pubertal development, and risk-taking behavior. J. Neurosci. 35 (18), 7226-7238. http://dx.doi.org/10.1523/jneurosci.4764-14.2015.

Brett, M., Anton, J., Valabregue, R., Poline, J., 2002. Region of interest analysis using an SPM toolbox Paper presented at In: Proceedings of the 8th International Conference on Functional Mapping of the Human Brain, Sendai, Japan.

Bromberg, U., Wiehler, A., Peters, J., 2015. Episodic future thinking is related to impulsive decision making in healthy adolescents. Child Dev. 86 (5), 1458-1468. http://dx.doi.org/10.1111/cdev.12390.

Bunge, S.A., Wright, S.B., 2007. Neurodevelopmental changes in working memory and cognitive control. Curr. Opin. Neurobiol. 17 (2), 243-250. http://dx.doi.org/ 10.1016/j.conb.2007.02.005

Carskadon, M.A., Acebo, C., 1993. A self-administered rating scale for pubertal development. J. Adolesc. Health 14 (3), 190-195. http://dx.doi.org/10.1016/1054139x(93)90004-9.

Cavanna, A.E., Trimble, M.R., 2006. The precuneus: a review of its functional anatomy and behavioural correlates. Brain 129, 564-583. http://dx.doi.org/10.1093/brain/ awl004.

Christakou, A., Brammer, M., Rubia, K., 2011. Maturation of limbic corticostriatal activation and connectivity associated with developmental changes in temporal discounting. Neuroimage 54 (2), 1344-1354. http://dx.doi.org/10.1016/ j.neuroimage.2010.08.067.

Clare, S., Helps, S., Sonuga-Barke, E.J., 2010. The quick delay questionnaire: a measure of delay aversion and discounting in adults. Atten. Deficit Hyperact. Disord. 2 (1), 43-48. http://dx.doi.org/10.1007/s12402-010-0020-4.

Cohen Kadosh, K., Luo, Q., de Burca, C., Sokunbi, M.O., Feng, J., Linden, D.E., Lau, J.Y., 2016. Using real-time fMRI to influence effective connectivity in the developing emotion regulation network. Neuroimage 125, 616-626. http://dx.doi.org/10.1016/ j.neuroimage.2015.09.070.

Crockett, L.J., Petersen, A.C., 1987. Pubertal status and psychosocial development: findings from the Early Adolescence Study. In: Lerner, R.M., Foch, T.T. (Eds.), Biological-Psychosocial Interactions in Early Adolescence. Lawrence Erlbaum Associates, Hillsdale, New Jersey, 173-188.

Crone, E.A., Dahl, R.E., 2012. Understanding adolescence as a period of social-affective engagement and goal flexibility. Nat. Rev. Neurosci. 13 (9), 636-650. http:// dx.doi.org/10.1038/nrn3313.

de Water, E., Braams, B.R., Crone, E.A., Peper, J.S., 2013. Pubertal maturation and sex steroids are related to alcohol use in adolescents. Horm. Behav. 63 (2), 392-397. http://dx.doi.org/10.1016/j.yhbeh.2012.11.018.

de Water, E., Cillessen, A.H.N., Scheres, A., 2014. Distinct age-related differences in temporal discounting and risk taking in adolescents and young adults. Child Dev. 85 (5), 1881-1897. http://dx.doi.org/10.1111/cdev.12245.

Demurie, E., Roeyers, H., Baeyens, D., Sonuga-Barke, E., 2012. Age-related changes in temporal reward discounting as a function of primary and secondary reinforcing properties (Doctoral thesis). In: Demurie, E. (Ed.), Sensitivity for reward in children with attention-deficit/hyperactivity disorder. Ghent University, Belgium, 101-121.

Demurie, E., Roeyers, H., Baeyens, D., Sonuga-Barke, E.J.S., 2012. Temporal discounting of monetary rewards in children and adolescents with ADHD and autism spectrum disorders. Dev. Sci. 15 (6), 791-800. http://dx.doi.org/10.1111/j.14677687.2012.01178.x.

Estle, S.J., Green, L., Myerson, J., Holt, D.D., 2007. Discounting of monetary and directly consumable rewards. Psychol. Sci. 18 (1), 58-63. http://dx.doi.org/10.1111/j.14679280.2007.01849.x.

Figner, B., Knoch, D., Johnson, E.J., Krosch, A.R., Lisanby, S.H., Fehr, E., Weber, E.U., 2010. Lateral prefrontal cortex and self-control in intertemporal choice. Nat. Neurosci. 13 (5), 538-539. http://dx.doi.org/10.1038/nn.2516.

Foerde, K., Figner, B., Doll, B.B., Woyke, I.C., Braun, E.K., Weber, E.U., Shohamy, D., 2016. Dopamine modulation of intertemporal decision-making: evidence from parkinson disease. J. Cogn. Neurosci. 28 (5), 657-667. http://dx.doi.org/10.1162/ jocn_a_00929.

Gianotti, L.R., Figner, B., Ebstein, R.P., Knoch, D., 2012. Why some people discount more than others: baseline activation in the dorsal PFC mediates the link between COMT genotype and impatient choice. Front. Neurosci. 6, 54. http://dx.doi.org/ 10.3389/fnins.2012.00054.

Hare, T.A., Camerer, C.F., Rangel, A., 2009. Self-control in decision-making involves modulation of the vmPFC valuation system. Science 324 (5927), 646-648. http:// dx.doi.org/10.1126/science.1168450.

Hare, T., Hakimi, S., Rangel, A., 2014. Activity in dlPFC and its effective connectivity to
vmPFC are associated with temporal discounting. Front. Neurosci. 8 (50). http:// dx.doi.org/10.3389/fnins.2014.00050.

Hare, T.A., Tottenham, N., Davidson, M.C., Glover, G.H., Casey, B.J., 2005. Contributions of amygdala and striatal activity in emotion regulation. Biol. Psychiatry 57 (6), 624-632. http://dx.doi.org/10.1016/j.biopsych.2004.12.038.

Hariri, A.R., Brown, S.M., Williamson, D.E., Flory, J.D., de Wit, H., Manuck, S.B., 2006 Preference for immediate over delayed rewards is associated with magnitude of ventral striatal activity. J. Neurosci. 26 (51), 13213-13217. http://dx.doi.org/ 10.1523/jneurosci.3446-06.2006.

Hsu, C.F., Benikos, N., Sonuga-Barke, E.J.S., 2015. Spontaneous activity in the waiting brain: a marker of impulsive choice in attention-deficit/hyperactivity disorder? Dev. Cogn. Neurosci. 12, 114-122. http://dx.doi.org/10.1016/j.den.2015.01.007.

Jackson, J.N.S., MacKillop, J., 2016. Attention deficit hyperactivity disorder and monetary delay discounting: a meta-analysis of case-control studies. Biol. Psychiatry: Cogn. Neurosci. Neuroimaging. http://dx.doi.org/10.1016/j.bpsc.2016.01.007.

Jimura, K., Myerson, J., Hilgard, J., Keighley, J., Braver, T.S., Green, L., 2011. Domain independence and stability in young and older adults' discounting of delayed rewards. Behav. Process. 87 (3), 253-259. http://dx.doi.org/10.1016/ j.beproc.2011.04.006.

Kable, J.W., Glimcher, P.W., 2007. The neural correlates of subjective value during intertemporal choice. Nat. Neurosci. 10 (12), 1625-1633. http://dx.doi.org/ $10.1038 / \mathrm{nn} 2007$.

Kable, J.W., Glimcher, P.W., 2010. An "As Soon As Possible" effect in human intertemporal decision making: behavioral evidence and neural mechanisms. J. Neurophysiol. 103 (5), 2513-2531. http://dx.doi.org/10.1152/jn.00177.2009.

Lansu, T.A.M., Cillessen, A.H.N., Karremans, J.C., 2012. Implicit associations with popularity in early adolescence: an approach-avoidance analysis. Dev. Psychol. 48 (1), 65-75. http://dx.doi.org/10.1037/a0025681.

Lemiere, J., Danckaerts, M., Van Hecke, W., Mehta, M.A., Peeters, R., Sunaert, S., Sonuga-Barke, E.J.S., 2012. Brain activation to cues predicting inescapable delay in adolescent Attention Deficit/Hyperactivity Disorder: an fMRI pilot study. Brain Res. 1450, 57-66. http://dx.doi.org/10.1016/j.brainres.2012.02.027.

Liu, X., Hairston, J., Schrier, M., Fan, J., 2011. Common and distinct networks underlying reward valence and processing stages: a meta-analysis of functional neuroimaging studies. Neurosci. Biobehav. Rev. 35 (5), 1219-1236. http:// dx.doi.org/10.1016/j.neubiorev.2010.12.012.

MacKillop, J., Amlung, M.T., Few, L.R., Ray, L.A., Sweet, L.H., Munafo, M.R., 2011. Delayed reward discounting and addictive behavior: a meta-analysis. Psychopharmacology 216 (3), 305-321. http://dx.doi.org/10.1007/s00213-0112229-0.

Masten, C.L., Eisenberger, N.I., Borofsky, L.A., Pfeifer, J.H., McNealy, K., Mazziotta, J.C., Dapretto, M., 2009. Neural correlates of social exclusion during adolescence: understanding the distress of peer rejection. Social. Cogn. Affect. Neurosci. 4 (2), 143-157. http://dx.doi.org/10.1093/scan/nsp007.

Mazur, J.E., Stellar, J.R., Waraczynski, M., 1987. Self-control choice with electrical stimulation of the brain as a reinforcer. Behav. Process. 15 (2-3), 143-153. http:// dx.doi.org/10.1016/0376-6357(87)90003-9.

McClure, S.M., Ericson, K.M., Laibson, D.I., Loewenstein, G., Cohen, J.D., 2007. Time discounting for primary rewards. J. Neurosci. 27 (21), 5796-5804. http:// dx.doi.org/10.1523/jneurosci.4246-06.2007.

McClure, S.M., Laibson, D.I., Loewenstein, G., Cohen, J.D., 2004. Separate neural systems value immediate and delayed monetary rewards. Science 306 (5695), 503-507. http://dx.doi.org/10.1126/science.1100907.

Mertens, J.R., Lu, Y.W., Parthasarathy, S., Moore, C., Weisner, C.M., 2003. Medical and psychiatric conditions of alcohol and drug treatment patients in an HMO: comparison with matched controls. Arch. Intern. Med. 163 (20), 2511-2517. http:// dx.doi.org/10.1001/archinte.163.20.2511.

Myerson, J., Green, L., Warusawitharana, M., 2001. Area under the curve as a measure of discounting. J. Exp. Anal. Behav. 76 (2), 235-243. http://dx.doi.org/10.1901/ jeab.2001.76-235.

Neef, N.A., Bicard, D.F., Endo, S., 2001. Assessment of impulsivity and the development of self-control in students with attention deficit hyperactivity disorder. J Appl. Behav. Anal. 34 (4), 397-408. http://dx.doi.org/10.1901/jaba.2001.34-397.

Nichols, T., Brett, M., Andersson, J., Wager, T., Poline, J.B., 2005. Valid conjunction inference with the minimum statistic. Neuroimage 25 (3), 653-660. http:// dx.doi.org/10.1016/j.neuroimage.2004.12.005.

Olson, E.A., Hooper, C.J., Collins, P., Luciana, M., 2007. Adolescents' performance on delay and probability discounting tasks: contributions of age, intelligence, executive functioning, and self-reported externalizing behavior. Pers. Individ. Differ. 43 (7), 1886-1897. http://dx.doi.org/10.1016/j.paid.2007.06.016.

Op de Macks, Z.A., Moor, B.G., Overgaauw, S., Guroglu, B., Dahl, R.E., Crone, E.A., 2011 Testosterone levels correspond with increased ventral striatum activation in response to monetary rewards in adolescents. Dev. Cogn. Neurosci. 1 (4), 506-516. http://dx.doi.org/10.1016/j.den.2011.06.003.

Patros, C.H., Alderson, R.M., Kasper, L.J., Tarle, S.J., Lea, S.E., Hudec, K.L., 2016. Choice-impulsivity in children and adolescents with attention-deficit/hyperactivity disorder (ADHD): a meta-analytic review. Clin. Psychol. Rev. 43, 162-174. http:// dx.doi.org/10.1016/j.cpr.2015.11.001.

Peper, J.S., Dahl, R.E., 2013. The teenage brain: surging hormones-brain-behavior interactions during puberty. Curr. Dir. Psychol. Sci. 22 (2), 134-139. http:// dx.doi.org/10.1177/0963721412473755.

Peper, J.S., Mandl, R.C.W., Braams, B.R., de Water, E., Heijboer, A.C., Koolschijn, P., Crone, E.A., 2013. Delay discounting and frontostriatal fiber tracts: a combined DTI and MTR study on impulsive choices in healthy young adults. Cereb. Cortex 23 (7), 1695-1702. http://dx.doi.org/10.1093/cercor/bhs163.

Peters, J., Buechel, C., 2009. Overlapping and distinct neural systems code for subjective 
value during intertemporal and risky decision making. J. Neurosci. 29 (50), 15727-15734. http://dx.doi.org/10.1523/jneurosci.3489-09.2009.

Peters, J., Buechel, C., 2010. Episodic future thinking reduces reward delay discounting through an enhancement of prefrontal-mediotemporal interactions. Neuron 66 (1), 138-148. http://dx.doi.org/10.1016/j.neuron.2010.03.026.

Petersen, A.C., Crockett, L., Richards, M., Boxer, A., 1988. A self-report measure of pubertal status- reliability, validity, and initial norms. J. Youth Adolesc. 17 (2), 117-133. http://dx.doi.org/10.1007/bf01537962.

Pfeifer, J.H., Masten, C.L., Moore, W.E., 3rd, Oswald, T.M., Mazziotta, J.C., Iacoboni, M., Dapretto, M., 2011. Entering adolescence: resistance to peer influence, risky behavior, and neural changes in emotion reactivity. Neuron 69 (5), 1029-1036. http://dx.doi.org/10.1016/j.neuron.2011.02.019.

Plichta, M.M., Vasic, N., Wolf, R.C., Lesch, K.P., Brummer, D., Jacob, C., Gron, G., 2009. Neural hyporesponsiveness and hyperresponsiveness during immediate and delayed reward processing in adult attention-deficit/hyperactivity disorder. Biol. Psychiatry 65 (1), 7-14. http://dx.doi.org/10.1016/j.biopsych.2008.07.008.

Poser, B.A., Versluis, M.J., Hoogduin, J.M., Norris, D.G., 2006. BOLD contrast sensitivity enhancement and artifact reduction with multiecho EPI: parallel-acquired inhomogeneity-desensitized fMRI. Magn. Reson. Med. 55 (6), 1227-1235. http:// dx.doi.org/10.1002/mrm.20900.

Qu, Y., Galvan, A., Fuligni, A.J., Lieberman, M.D., Telzer, E.H., 2015. Longitudinal changes in prefrontal cortex activation underlie declines in adolescent risk taking. J. Neurosci. 35 (32), 11308-11314. http://dx.doi.org/10.1523/jneurosci.155315.2015.

R Core Team, 2013. R: A language and environment for statistical computing. $\mathrm{R}$ Foundation for Statistical Computing, Vienna, Austria. ISBN 3-900051-07-0, URL: 〈http://www.R-project.org/〉.

Reynolds, B., 2006. The Experiential Discounting Task is sensitive to cigarette-smoking status and correlates with a measure of delay discounting. Behav. Pharmacol. 17 (2), 133-142. http://dx.doi.org/10.1097/01.fbp.0000190684.77360.c0.

Ripke, S., Hubner, T., Mennigen, E., Muller, K.U., Rodehacke, S., Schmidt, D., Smolka, M.N., 2012. Reward processing and intertemporal decision making in adults and adolescents: the role of impulsivity and decision consistency. Brain Res. 1478, 36-47 . http://dx.doi.org/10.1016/j.brainres.2012.08.034.

Scheres, A., de Water, E., Mies, G.W., 2013. The neural correlates of temporal reward discounting. Wiley Interdiscip. Rev.-Cogn. Sci. 4 (5), 523-545. http://dx.doi.org/ $10.1002 /$ wcs. 1246 .

Scheres, A., Dijkstra, M., Ainslie, E., Balkan, J., Reynolds, B., Sonuga-Barke, E., Castellanos, F.X., 2006. Temporal and probabilistic discounting of rewards in children and adolescents: effects of age and ADHD symptoms. Neuropsychologia 44 (11), 2092-2103. http://dx.doi.org/10.1016/j.neuropsychologia.2005.10.012.

Scheres, A., Tontsch, C., Thoeny, A.L., Kaczkurkin, A., 2010. Temporal reward discounting in attention-deficit/hyperactivity disorder: the contribution of symptom domains, reward magnitude, and session length. Biol. Psychiatry 67 (7), 641-648. http://dx.doi.org/10.1016/j.biopsych.2009.10.033.

Scheres, A., Tontsch, C., Thoeny, A.L., Sumiya, M., 2014. Temporal reward discounting in children, adolescents, and emerging adults during an experiential task. Front. Psychol. 5. http://dx.doi.org/10.3389/fpsyg.2014.00711.
Sescousse, G., Caldu, X., Segura, B., Dreher, J.C., 2013. Processing of primary and secondary rewards: a quantitative meta-analysis and review of human functional neuroimaging studies. Neurosci. Biobehav. Rev. 37 (4), 681-696. http://dx.doi.org/ 10.1016/j.neubiorev.2013.02.002.

Singmann, H., Bolker, B., \& Westfall, J., 2015. afex: Analysis of Factorial Experiments. 〈http://CRAN.R-project.org/package=afex $\rangle$.

Stanger, C., Elton, A., Ryan, S.R., James, G.A., Budney, A.J., Kilts, C.D., 2013. Neuroeconomics and adolescent substance abuse: individual differences in neural networks and delay discounting. J. Am. Acad. Child Adolesc. Psychiatry 52 (7), 747-755. http://dx.doi.org/10.1016/j.jaac.2013.04.013.

Steinberg, L., 2008. A social neuroscience perspective on adolescent risk-taking. Dev. Rev. 28 (1), 78-106. http://dx.doi.org/10.1016/j.dr.2007.08.002.

Steinberg, L., Graham, S., O'Brien, L., Woolard, J., Cauffman, E., Banich, M., 2009. Age differences in future orientation and delay discounting. Child Dev. 80 (1), 28-44. http://dx.doi.org/10.1111/j.1467-8624.2008.01244.x.

van den Bos, W., McClure, S.M., 2013. Towards a general model of temporal discounting. J. Exp. Anal. Behav. 99 (1), 58-73. http://dx.doi.org/10.1002/jeab.6.

van den Bos, W., Rodriguez, C.A., Schweitzer, J.B., McClure, S.M., 2015. Adolescent impatience decreases with increased frontostriatal connectivity. Proc. Natl. Acad. Sci. USA 112 (29), E3765-E3774. http://dx.doi.org/10.1073/pnas.1423095112.

van Duijvenvoorde, A.C.K., Huizenga, H.M., Somerville, L.H., Delgado, M.R., Powers, A., Weeda, W.D., Figner, B., 2015. Neural correlates of expected risks and returns in risky choice across development. J. Neurosci. 35 (4), 1549-1560. http://dx.doi.org/ 10.1523/jneurosci.1924-14.2015.

Weber, B.J., Huettel, S.A., 2008. The neural substrates of probabilistic and intertemporal decision making. Brain Res. 1234, 104-115. http://dx.doi.org/10.1016/ j.brainres.2008.07.10S.

Wechsler, D., 1991. Manual for the Wechsler Intelligence Scale for Children-Third Edition (WISC-III). The Psychological Corporation, San Antonio, TX.

Wesley, M.J., Bickel, W.K., 2014. Remember the future II: meta-analyses and functional overlap of working memory and delay discounting. Biol. Psychiatry 75 (6), 435-448. http://dx.doi.org/10.1016/j.biopsych.2013.08.008.

White, S.F., Clanton, R., Brislin, S.J., Meffert, H., Hwang, S., Sinclair, S., Blair, R.J.R., 2014. Temporal discounting and conduct disorder in adolescents. J. Pers. Disord. 28 (1), 5-18.

Wilbertz, G., Trueg, A., Sonuga-Barke, E.J.S., Blechert, J., Philipsen, A., van Elst, L.T., 2013. Neural and psychophysiological markers of delay aversion in attention-deficit hyperactivity disorder. J. Abnorm. Psychol. 122 (2), 566-572. http://dx.doi.org/ $10.1037 / \mathrm{a} 0031924$.

Wood, P., 2009. Salivary steroid assays - research or routine? Ann. Clin. Biochem. 46 (Pt 3), 183-196. http://dx.doi.org/10.1258/acb.2008.008208.

Yarkoni, T., Poldrack, R.A., Nichols, T.E., Van Essen, D.C., Wager, T.D., 2011. Largescale automated synthesis of human functional neuroimaging data. Nat. Methods 8 (8), 665-U695. http://dx.doi.org/10.1038/nmeth.1635.

Zauberman, G., Kyu Kim, B., Malkoc, S.A., Bettman, J.R., 2008. Discounting time and time discounting: subjective time perception and intertemporal preferences. J. Mark. Res. 46, 543-556. 